\title{
Design and implementation of IOT based Gas detection \& fire extinguishing System
}

\author{
Mendi Manoj Kumar ${ }^{1}$, Vendrapu Appala Naidu ${ }^{2}$, Sunkarapalli Mohit ${ }^{3}$, Patnayakuni Avyakth $^{4}$, \\ Dr. B V S Acharyulu ${ }^{5}$ \\ ${ }^{1}$ Student,Department of EEE, Lendi Institute of engineering \& technology, INDIA, \\ manojkumarmendi@gmail.com, \\ ${ }^{2}$ Student,Department of EEE, Lendi Institute of engineering \& technology, INDIA, \\ naiduvendrapu234@gail.com, \\ ${ }^{3}$ Student,Department of EEE, Lendi Institute of engineering \& technology, INDIA,s.mohith222@ gmail.com, \\ ${ }^{4}$ Student,Department of EEE, Lendi Institute of engineering \& technology, INDIA, Avyakthpatnaik@ gmail.com, \\ ${ }^{5}$ Associate professor, Department of EEE, Lendi Institute of engineering \& technology, INDIA, \\ acharyulu201@yahoo.com
}

\begin{abstract}
The protection of houses from the fire is a major challenge due to the growing population and growth of number of buildings. The gas detection and circuit breaking definitely will be the first step to reduce this problem. Furthermore it is more useful to provide much more security to the mines with less cost and without sacrificing the quality of equipment. The project presents a new way for securing the domestic and industrial buildings from causing the fire and providing the information to the user as soon as it detects the gas and fire. It also uses the IOT system, when identified the fire and informing the user through an application.As soon as the gas is detected the module turns off the nearby circuit board using H-bridge, so that nearby electrical devices would not cause any type of the causes for the fire. The usage of the low power devices would not consume much of the power. It also turns on the ventilator fan for exhausting of the gases.Using this kind of modules would enhance the opportunities for the safety of the buildings and saving more lives.In this paper we have designed Internet of Things based system which enables the early detection of fire and gas leaks.
\end{abstract}

Key words: Micro controllers,Arduino,L298 module, Flame detection, Gas detection sensor, IOT.

\section{INTRODUCTION}

In the growing population and also the growth of the density of population the usage of the gas and also fire accidents occurred by it is increasing year by year. This project makes the domestic use of the gas (lpg) more secure and safe. It enables the use of lpg or other gases in a safe way. The help of technology is needed to provide early warning alerts in order to ensure that enough time is available to prevent potential dangers. In this study, gas leakage detection systems have been highlighted along with troubleshooting directives. A warning system has been developed based on ESP (Wi-Fi) network. The system detects the presence of natural gases such as Methane and LPG. Whenever a gas leakage occurs, the sensors used in the circuit will detect it, the ESP modem will be activated and a notification alert to the user will be transmitted by a customized dashboard. The system provides a real-time notification, allowing immediate implementation of health and safety measures and increasing the available time span to overcome the situation.

ARDUINO and its specifications also along with the components in ARDUINO such as Transistor, Pushbuttons, Diode, Capacitors. Arduino was designed to help you get things done. To make that happen, we kept the background material on programming and electronics to a minimum .ARDUINO programming and its interfacing with the peripherals, external futures, pins and signals, internal architecture, programe memory organization, data memory organization, system lock, reset, programe branching instructions, unconditional jumps, conditional jumping. ARDUINO peripheral interfacing and basic programming, simple applications of LCD, Digital input and output, parallel ports, communication peripherals, architecture and developing of the basic projects. Most code is written in $\mathrm{C}$ but you may occasionally need to write a subroutine in assembly language to perform an operation that cannot be written out directly in C. Two examples are operations that require bitwise rotations rather than shifts and calculations that can be done more efficiently by exploiting special instructions of the CPU, such as binary-coded decimal arithmetic. Intrinsic functions often avoid the need for assembly language but not always. .Wireless networks are integral to IOT systems. Wireless network connections simplify installation and operation of wireless networks. However, wireless networks introduce some important problems and restrictions. Radio communication requires more power than does wired communication. Some of the wireless networks used in today's IoT devices were designed for other purposes, such as telephony and multimedia. As a result, they are not optimized 
for event-driven communication and consume significant amounts of power in the communications protocol. The design space for IoT devices is very different from that for mobile or cloud processors. Both mobile and cloud systems require very large chips. IoT devices should operate at extremely low power levels but often not operate continuously.[1-4]

Internet has become an important part of human's social life and educational life without which they are just helpless. The Internet of things (Iot) devices not only controls but also monitors the electronic, electrical and various mechanical systems which are used in various types of infrastructures. These devices which are connected to the cloud server are controlled by a single user (also known as admin) which are again transmitted or notified to all the authorized user connected to that network. Various electronics and electrical devices are connected and controlled remotely through different network infrastructures. Web browser present in laptop or smart phone or any other smart technique through which we can operate switches, simply removes the hassle of manually operating a switch[5-8].

The sensor is connected to the input of the arduino with the help of connecting cables or jumper cables. Further the circuit goes towards output where the buzzer is connected. If we differ the delay value of the buzzer then we get a variation in the buzzer sound. This can be applicable to detect various gases. Arduino is specially used in this design as it is easy to use in the circuit and the program upload is also very simple. That is why arduino is used. It is provided with $5 \mathrm{v}$ dc , which is a advantage that it won't require much power and is easy to install. It's SMS technique can also be used with GSM technology to send messages to emergency services. We can also use it to detect various other gases other than LPG and CNG. It will be helpful in public places like Mall's, hospital's, hotel's etc. In such places there is a huge rush of people, women and small children. Liquefied petroleum fuel (LPG) is the most vital phase of every day human life, however the protection of human existence is required for the gasoline explosion. This methodology is used to shield human life. In this, the MQ2 sensor is used to realize fuel leaks in the home. If the fuel leak is detected automatically, it will ship an SMS to the user. Wifi is one of the most used networks in the world. [9-10]

Internet of Things aim towards making life simpler by automating every small task around us. As much is IoT helping in automating tasks, the benefits of IoT can also be extended for enhancing the existing safety standards. Safety, the elementary concern of any project, has not been left untouched by IoT. Gas Leakages in open or closed areas can prove to be dangerous and lethal. The traditional Gas Leakage Detector Systems though have great precision, fail to acknowledge a few factors in the field of alerting the people about the leakage. Therefore we have used the IoT technology to make a Gas Leakage Detector for society which having Smart Alerting techniques involving sending text message to the concerned authority [11-13]
Prevention of fire and fire risk level control difficulty are increased day by day. Fire-fighting and monitoring situations are very serious today. Public security keep on insisting in increase of technology in firefighting and monitoring. They give special attention to improve the science and technology in resisting fire disasters. They are concerned about the application of new technology such as IoT and wireless sensor network in fire-fighting and monitoring field. IoT is very suitable for fire-fighting with wide scope along with wireless sensor network (WSN). IoT has high degree of intelligence for maintaining many product categories, quantities, complex fire danger factors and large range of equipments for fire monitoring and fighting[14-15].

Internet of Things is responsible for completing tasks while one is involving in other activities. IoT based Smart Home Management,The IoT based automation system is totally different from other wireless automation system since, it can be operating from other wireless automation system. [16].

\section{SYSTEM ARCHITECTURE:}

\subsection{COMPONENTS OF SYSTEM ARCHITECTURE}

$>$ Node MCU

Micro controllers

$>$ Arduino

$>$ L298 module(H-bridge)

$>$ IR Flame sensor

MQ2 Gas sensor

$>$ Exhaust fan

$>$ Buzzer

BLYNK software.

\subsection{PROPOSED MODEL}

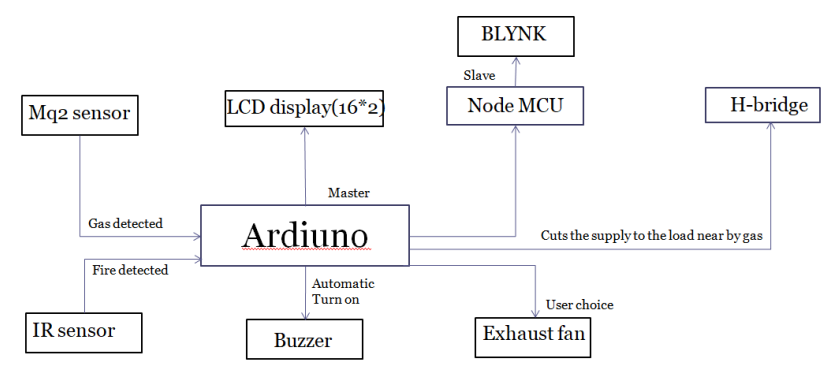

Figure-1:proposed model

The figure-1 shows how the system works. After taking the inputs from the sensors the Arduino starts working by communicating with the peripherals connected to it. The microcontroller acts as the master for all the devices connected to it. Hence the user gets the information via internet using the node mcu. The exhaust fan will be turned on automatically for the particular level of gas and also user can turn on exhaust fan remotely. the level of the gas detected will always be shown in the users handset which allows him to have the continues information of the gas level at the particular place.the H-bridge here acts as the relay and turns off the load of nearby circuit board which is a cause for the fire in the domestic places. The turning ON or OFF of thr exhaust 
fan is in the user choice. As we connected the device with the internet it can be operated world wide. Also we set the caution for the level of gas that is the exhaust fan overrides the IOT and it turns ON automatically for the particular level of gas. The level can be changed by changing the programe dumped in to the ARDUINO board. By this option the user can be protected more efficiently and also the exhaust can be used for forcing out the hot gases while cooking and other porposes. The messages can be seen on the LCD display.

\section{3 . INTERFACING OF THE ARDUINO BOARD WITH PHERPHERALS:}

\section{1 : INTERFACING OF NODE MCU WITH ARDUINO}

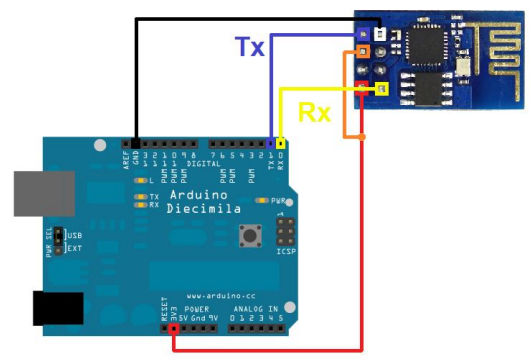

Figure-2:interfacing of arduino with node $M C U$

The arduino interfaces with the nodeMCU in order send the alerts to the user. The figure -2 shows the interfacing of the ARDUINO with node MCU.The node MCU and the Arduino will be communicating with each other about the value of the gases and also the detection of the fire through the TX/RX pins.Here the arduino will be acting as the master and the node MCU will be acting as the slave. We are giving some Wi-Fi name and password details in the NodeMCU programming. Then we have to match the same credentials to our mobile for the connection of internet to the NodeMCU. Hence nodeMCU plays a vital role in the hardware.

\section{2 : INTERFACING OF MQ2 GAS SENSOR WITH ARDUINO}

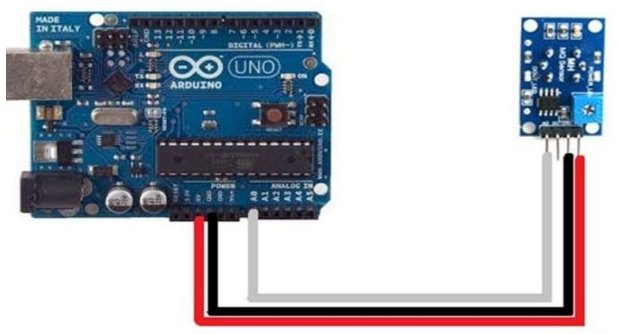

Figure-3:interfacing of arduino with $\mathrm{mQ2}$ gas sensor

The arduino interfaces with the MQ2 gas sensor in order to detect the gas that was leaked.The MQ2 gas sensor sends the signal to the arduino in the form of voltage i.e $(\mathrm{mV})$. The figure -3 shows the interfacing of the ARDUINO with MQ2 gas sensor. The arduino then turns off the load connected the circuit board, also turns on the buzzer and send an alert to the user through Internet by means of node MCU. The resistance of the sensor is different depending on the type of the gas. The smoke sensor has a built-in potentiometer that allows you to adjust the sensor sensitivity according to how accurate you want to detect gas.

\section{3: INTERFACING OF FLAME SENSOR AND BUZZER WITH ARDUINO}

In the same way as gas detection sensor acted,the IR flame sensor detects the flame and alerts the user and gives an option to the user weather to turn on the exhaust fan or not. But the buzzer is the default setting when the gas/fire is detected the buzzer automatically turns on.

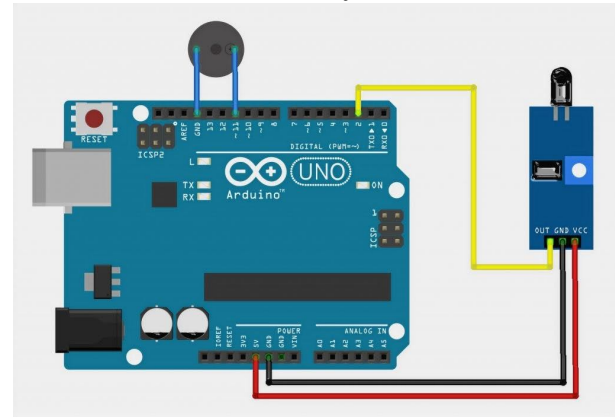

Figure-4:Flame sensor and Buzzer connected to ARDUINO

The figure -4 shows the interfacing of the IR flame sensor with the ARDUINO board.

\section{4 : INTERFACING OF H-BRIDGE WITH ARDUINO}

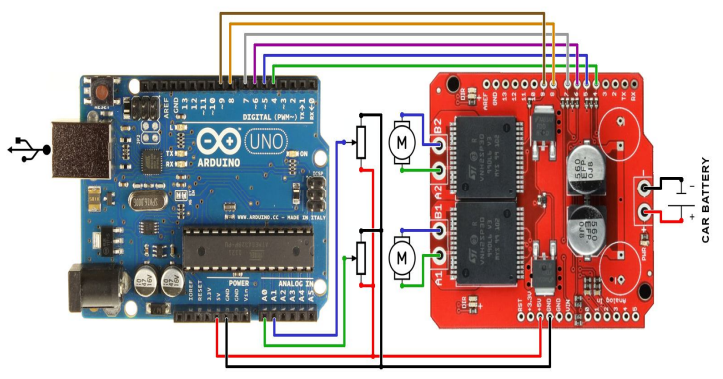

Figure-5:Arduino connected to the H-bridge

The H-bridge here acts to turn off the load connected to the circuit board or in other words it acts as the relay. The $\mathrm{H}$-bridge communicates with the Arduino with voltage (i.e the Arduino sends the signal in the form of voltage as milliVolts which in turn amplified by the h-bridge to change polarity). An "H-Bridge" is simply an arrangement of switching the polarity of the voltage applied to a DC motor, thus controlling its direction of rotation. The figure -5 shows the interfacing of the ARDUINO with the H-bridge. The L298N actually contains two complete H-Bridge circuits, so it is capable of driving a pair of DC motors. This makes it ideal 
for robotic projects, as most robots have either two or four powered wheels. The L298N can also be used to drive a single stepper motor.

\section{5 :INTERFACING OF LCD DISPLAY AND EXHAUST WITH ARDUINO}

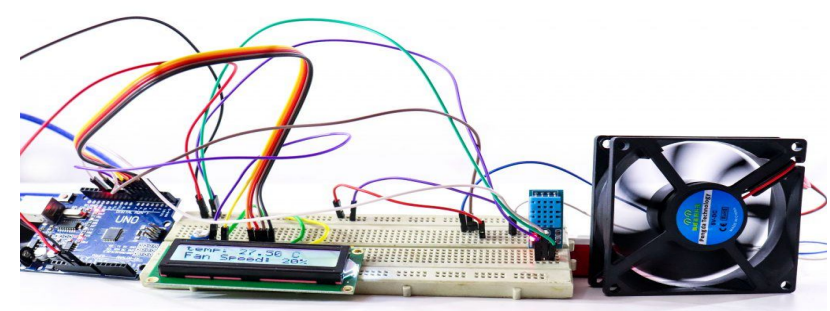

\section{Figure-6:Arduino connected with the exhaust fan and also the LCD display}

As soon as the gas/flame is detected by the sensors the level of the gas detected will be displayed on the LCD display and on the user choice the exhaust will be turned on. .The figure -6 shows the interfacing of the ARDUINO with the LCD display and exhaust fan. As the hardware is connected to the internet directly user can turn on/off the exhaust fan when ever he needs. The detection of the flame is also displayed on the LCD screen and at the same time it sends and alert message to the user. Basing on the gas level detected the user can turn on the exhaust or if gas level is high it automatically turns on exhaust and turns off load near by it. The LCD display is interfaced with the Arduino by using the pwm pins of the arduino. We will use just 6 digital input pins from the Arduino Board. The LCD's registers from D4 to D7 will be connected to Arduino's digital pins from 4 to 7 . The Enable pin will be connected to pin number 2 and the RS pin will be connected to pin number 12 . The second pin is the VCC which we connect the 5 volts pin on the Arduino Board.

\section{3..6 : INTERFACING OF BLYNK SOFTWARE WITH ARDUINO}

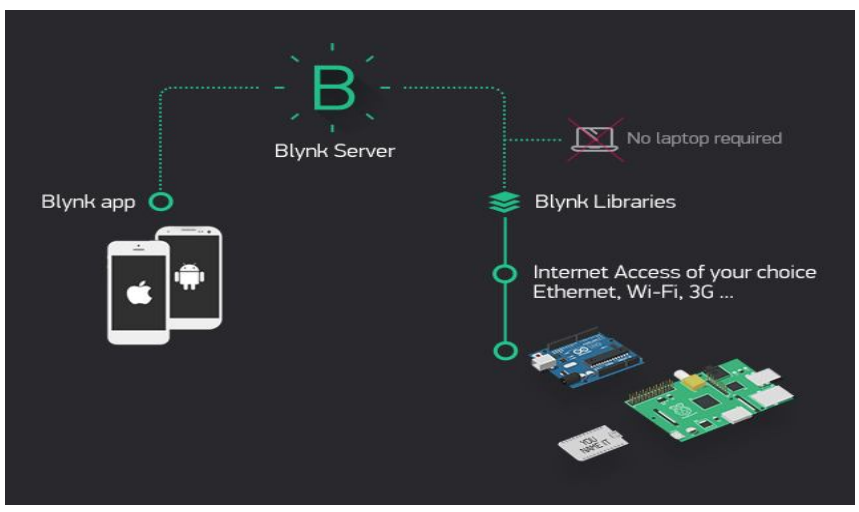

Figure-7:BLYNK software used to control the exhaust fan
NodeMCU is an open source and LUA programming language based firmware developed for ESP8266 Wi-Fi chip. ESP8266 has very low cost and high features which makes it an ideal module for Internet of Things (IOT). The figure-7 shows how the BLYNK software is connected to the node MCU. It can be used in any application that requires it to connect a device to local network or internet.

BLYNK combines a cloud platform with applications that put things, people, and data at the heart of business operations. BLYNK was designed for the Internet of Things. It can control hardware remotely, it can display sensor data, it can store data, visualize it and do many other cool thingsUser can operate the exhaust fan at any location all over the world from the position he was. On turning on the exhaust fan the gases detected can be flood out from the contained zone.

\section{7 : JUMPER WIRES USED FOR CONNECTING PHERIPHERALS}

The jump wires shown in figure -8 (also known as jumper, jumper wire, jumper cable, DuPont wire or cable) is an electrical wire, or group of them in a cable, with a connector or pin at each end (or sometimes without them simply "tinned"), which is normally used to interconnect the components of a breadboard or other prototype or test circuit, internally or with other equipment or components, without soldering.

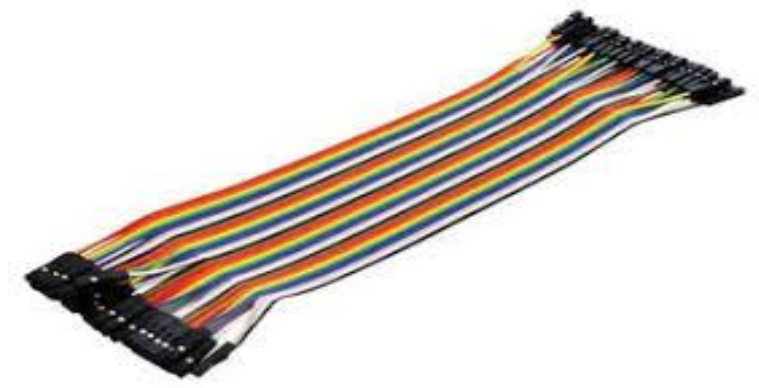

Figure-8:jumper wires

Individual jump wires are fitted by inserting their "end connectors" into the slots provided in a breadboard, the header connector of a circuit board, or a piece of test equipment.

\subsection{HARDWARE SYSTEM}

The pheripherals that was explained in the interfacing was connected as shown above using the jumper wires.All the anlouge pins of the nodeMCU, MQ2 gas sensor, IR flame sensor are connected to the analog pin of Arduino and the grounds are connected together at a same point of all components. The RX/TX pins are connected to the $(0,1)$ of the Arduino. The LCD display is connected in a way that the pins $(1,3,5)$ are grounded and the pin 2 is connected to source and the pin 4 is connected to the pin 11 of the Arduino and pin 6 is connected to the pin 12 of the arduino and digital 
pins $(4,5,6,7)$ are connected to the pwm pins of Arduino in the order of $(5,4,3,2)$. Simply changing the frequency of the voltage sent to the piezo and it will start generating sounds by changing shape very quickly.

\section{RESULTS AND DISCUSSIONS}

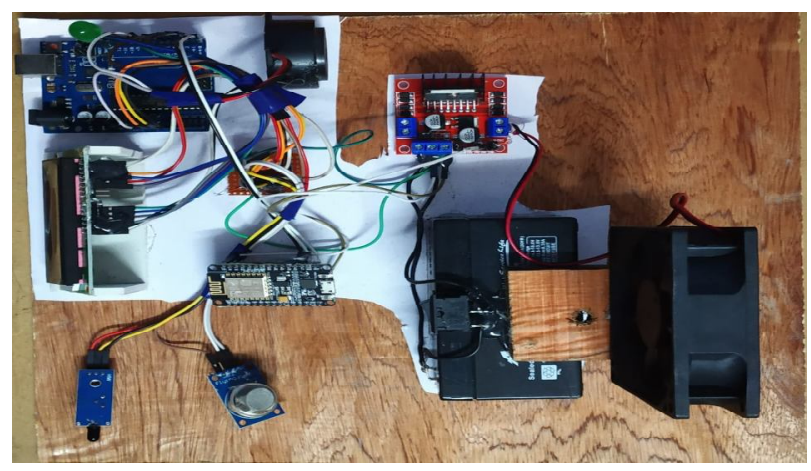

Figure-9:Hardware implementation

Table-1:Table showing the state off load

\begin{tabular}{|c|c|c|}
\hline SI no & Mode of operation of sensor & Load state \\
\hline 1 & MQ2 gas sensor ON /DETECT GAS & OFF \\
\hline $\mathbf{2}$ & IR flame sensor ON /DETECT FIRE & OFF \\
\hline
\end{tabular}

The hardware shown in figure- 9 is working at the domestical environment perfectly and also the gas detection and fire detection are done. The exhaust fan connected to the internet is also working perfectly. The table -1 shows the state of the load at the sensor operating conditions.

Table-2:Table showing the state of the exhaust fan

\begin{tabular}{|l|l|l|}
\hline Slno & State of sensor & State of exhaust \\
\hline 1 & $\begin{array}{l}\text { MQ2 gas sensor in active } \\
\text { state detecting gas. }\end{array}$ & ON \\
\hline 2 & $\begin{array}{l}\text { IR flame sensor in active state } \\
\text { detecting fire }\end{array}$ & ON \\
\hline
\end{tabular}

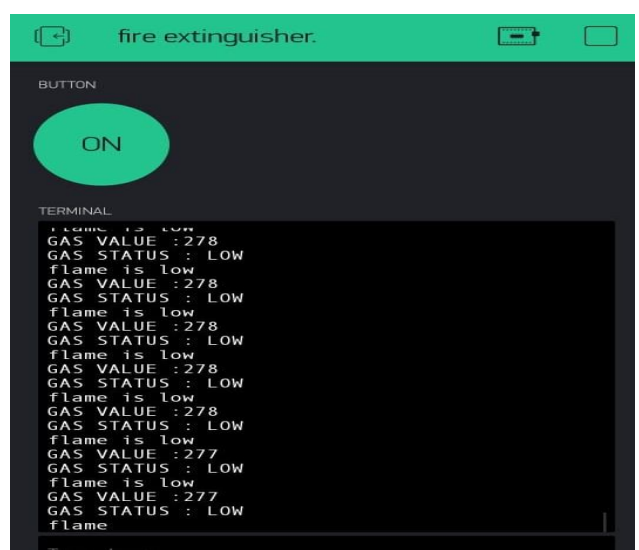

Figure -10:turning the exhaust ON from the BLYNK in hand set.

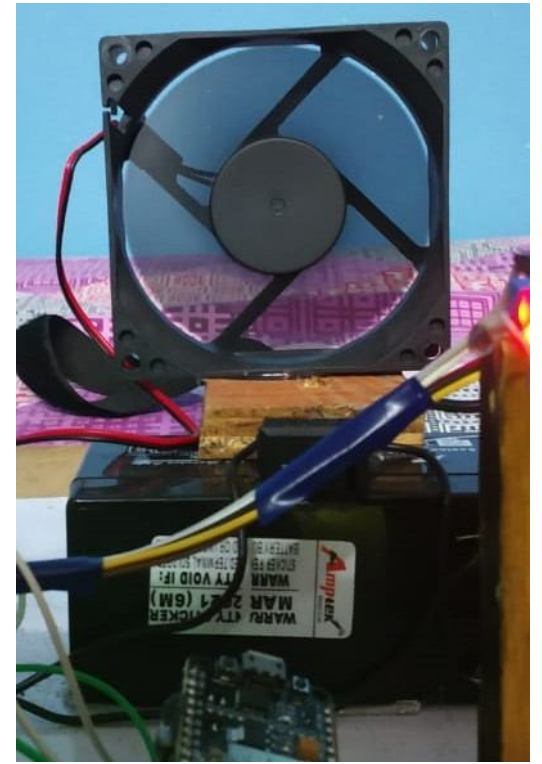

Figure-11:exhaust fan turned ON by using IOT

the figure-10 shows the results from the hand set connected to the hardware and the figure-11 shows the rotating of exhaust by it. The table -2 shows the state of the exhaust weather it is in the ON state or it is in the OFF state.

\section{CONCLUSION}

The project presents a new way for securing the domestic and industrial buildings from causing the fire and providing the information to the user as soon as it detects the gas and fire. It also uses the IOT system, when identified the fire and informing the user through an applicationHence the software results and hardware results are identical and the the designed hardware is working perfectly in the domestic environment. Advanced developments can be done to this project by connecting it to the house.

\section{REFERENCES}

[1]. Subrata Ghoshal(2010),"Microcontroller : Internals, Instructions, Programming and Interfacing",Pearson Education.

[2]. Davies J H (2011),"Microcontroller Basics”, Elsevier publications.

[3]. Dimitrios Serpanos, Marilyn Wolf(2018),'Internet of Things: The IOT Landscape", Springer International.

[4]. T. Begum, M. S. Hossain, M. B. Uddin and M. S. H. Chowdhury, "Design and development of activation and monitoring of home automation system via SMS through microcontroller" , Proc. 2009 4th International Conference on Computers and Devices for Communication (CODEC 2009), pp. 1-3.

[5]. ShopanDey, AyonRoy and SandipDas,"Home Automation Using Internet of Thing”, IRJET, 2(3) 
Mendi Manoj Kumar et al., International Journal of Emerging Trends in Engineering Research, 9(7), July 2021, 944 - 949

(2016),1965-1970. International Journal of Pure and Applied Mathematics Special Issue 7763.

[6]. Vishwateja Mudiam Reddy, Naresh Vinay, TapanPokharna and Shashank Shiva Kumar Jha, "Internet of Things Enabled Smart Switch", Thirteenth International Conference on Wireless and Optical Communications Networks (WOCN), Hyderabad, (2016),1-4 4.

[7]. Warsuzarina Mat Jubadi and NormaziahZulkifli, "Programmable Infrared Accessory Light Switch", International Conference on Intelligent and Advanced Systems, Kuala Lumpur,(2007), 1130-1134. 5.

[8]. Shih-Pang Tseng, Bo-Rong Li, Jun-Long Pan, and Chia-Ju Lin,"An Application of Internet of Things with Motion Sensing on Smart House”, International Conference on Orange Technologies, Xian, (2014), 65-68.

[9]. Mr. Parag Naik, Mr. Ashish Sambare, Ms. Dishanki Panpaliya,Ms. Harshali Janbandhu "International Conference On Innovations And Development For Future Smart Cities" (Icidfsc-17) ,March 2017.

[10].T. Thaker, "ESP8266 based implementation of wireless sensor network with Linux based web-server", Symposium on Colossal Data Analysis and Networking (CDAN), 2016.

[11]. MS Kasar, Rupali Dhaygude, Snehal Godse and Sneha Gurgule, "Automatic lpg gas booking and detection system", International Journal of Advanced Research in Electrical Electronics and Instrumentation Engineering, $p p$. 2278-8875, 2016.

[12]. Abid Khan, Neju K Prince, Shailendra Kumar Dewangan and Praveen Singh Rathore, "Gsm based automatic lpg ordering system with leakage alert", IJRET: International Journal of Research in Engineering and Technology, vol. 3, 2014.

[13]. Alan Macker, Anil Kumar Shukla, Sagarika Dey and JyothiAgarwal, "Arduino based lpg gas monitoring automatic cylinder booking with alert system", 2018 2nd International Conference on Trends in Electronics and Informatics (ICOEI), pp. 1209-1212, 2018.

[14]. Prabhakar S Varma and K. Jayavel, "Gas leakage detection and smart alerting and prediction using iot", 2017

2nd International Conference on Computing and Communications Technologies (ICCCT), pp. 327-333, Feb 2017.

[15]. Y. Y. Li, "Investigation of kitchen fire accidents in the united states", Fire Science and Thechnology, vol. 32, pp. 651, June 2013.

[16]. M.Prabu , Md Kazmi Alam, Sagar Soni , Karan Singh, "Smart Home Management Using IoT" International Journal of Emerging Technologies in Engineering Research (IJETER) Volume 6, Issue 11, November (2018),ISSN: 2454-6410 . 